\title{
Certain Properties of a Generalized Class of Analytic Functions Involving Some Convolution Operator
}

\author{
Faroze Ahmad Malik ${ }^{1, *}$, Nusrat Ahmed Dar $^{2}$ and Chitaranjan Sharma ${ }^{3}$ \\ ${ }^{1}$ Department of Mathematics, Government Holkar (Model, Autonomous) Science College, \\ Indore (M.P.)-452017, India \\ e-mail: malikferooze@gmail.com
}

2 Department of Mathematics, University of Kashmir, South Campus, Anantnag-192101, Jammu and Kashmir, India e-mail: nusratdar11@gmail.com

${ }^{3}$ Department of Mathematics, Government Holkar (Model, Autonomous) Science College, Indore (M.P.)-452017, India e-mail: drcsharma3@gmail.com

\begin{abstract}
We use the concept of convolution to introduce and study the properties of a unified family $\mathcal{T U M}_{\gamma}(g, b, k, \alpha),(0 \leq \gamma \leq 1, k \geq 0)$, consisting of uniformly $k$-starlike and $k$-convex functions of complex order $b \in \mathbb{C} \backslash\{0\}$ and type $\alpha \in[0,1)$. The family $\mathcal{T U M}_{\gamma}(g, b, k, \alpha)$ is a generalization of several other families of analytic functions available in literature. Apart from discussing the coefficient bounds, sharp radii estimates, extreme points and the subordination theorem for this family, we settle down the Silverman's conjecture for integral means inequality. Moreover, invariance of this family under certain well-known integral operators is also established in this paper. Some previously known results are obtained as special cases.
\end{abstract}

\section{Introduction}

Let $\mathcal{H}:=\mathcal{H}(\mathbb{D})$ be the collection of all functions $f(z)$ that are analytic in the open unit disc $\mathbb{D}:=\{z \in \mathbb{C}:|z|<1\}$, and let $\mathcal{A}$ denote the class of normalized analytic

Received: April 11, 2021; Accepted: May 18, 2021

2020 Mathematics Subject Classification: 30C45, 30C80.

Keywords and phrases: convolution, uniform $k$-starlikeness (convexity), functions of complex order, integral means, subordination.

${ }^{*}$ Corresponding author 
functions $f(z)$ of the form

$$
f(z)=z+\sum_{n=2}^{\infty} a_{n} z^{n} .
$$

A function $f \in \mathcal{A}$ is said to be uniformly $k$-starlike of order $\alpha$ if it satisfies

$$
\Re\left\{\frac{z f^{\prime}(z)}{f(z)}-\alpha\right\}>k\left|\frac{z f^{\prime}(z)}{f(z)}-1\right|,
$$

where $0 \leq \alpha<1, k \geq 0$. Let us denote the class of all such functions by $\mathcal{U S}(\alpha, k)$. Similarly, $f(z)$ is said to belong to the class of uniformly $k$-convex functions of order $\alpha, \mathcal{U C}(\alpha, k)$, if it satisfies

$$
\Re\left\{1+\frac{z f^{\prime \prime}(z)}{f^{\prime}(z)}-\alpha\right\}>k\left|\frac{z f^{\prime \prime}(z)}{f^{\prime}(z)}\right|,
$$

where $0 \leq \alpha<1$ and $k \geq 0$. Geometrically, $f \in \mathcal{U S}(\alpha, k)$ (or $\mathcal{U C}(\alpha, k)$ ) if and only if all the values taken by the expression $z f^{\prime}(z) / f(z)$ (or $\left.1+z f^{\prime \prime}(z) / f^{\prime}(z)\right)$ lie in the conic domain $\triangle_{\alpha, k}$ included in the right-half plane $\Re(w)>(\alpha+k) /(1+k)$ and is defined as

$$
\triangle_{\alpha, k}:=\left\{u+i v \in \mathbb{C}:(u-\alpha)^{2}>k^{2}\left((u-1)^{2}+v^{2}\right)\right\} .
$$

Note that $\triangle_{\alpha, k}$ represents (i) the right-half plane $\Re(w)>\alpha$ for $k=0$, (ii) a hyperbolic domain for $0<k<1$, (iii) a parabolic domain for $k=1$, and (iv) an elliptic domain for $k>1$. A pictorial representation of the domain $\triangle_{\alpha, k}$ is given in Figure 1, where we have taken $\alpha=1 / 2$ and $k \in\{0,0.5,1,1.5\}$. Observe that the conic regions are symmetric about the $u$-axis. For further details, see Kanas and Wiśniowska 24,25].

The classes $\mathcal{U S}(\alpha, k)$ and $\mathcal{U C}(\alpha, k)$ also generalize various previously defined function classes. For example $\mathcal{U S}(\alpha, 0)=\mathcal{S}^{*}(\alpha)$ and $\mathcal{U C}(\alpha, 0)=\mathcal{C}(\alpha)$ are, respectively, the families of starlike and convex functions of order $\alpha$, which were introduced by Robertson [39]. Also $\mathcal{U S}^{*}(0,0)=\mathcal{S}^{*}$ and $\mathcal{U C}(0,0)=\mathcal{C}$ are the well-known classes of starlike and convex functions. Further, the families $\mathcal{U S}(0,1)=\mathcal{U S}$ and $\mathcal{U C}(0,1)=\mathcal{U C}$ were introduced and investigated by Goodman 18, 19 and Rönning 40,41]. For further details, see [13 and references therein. 


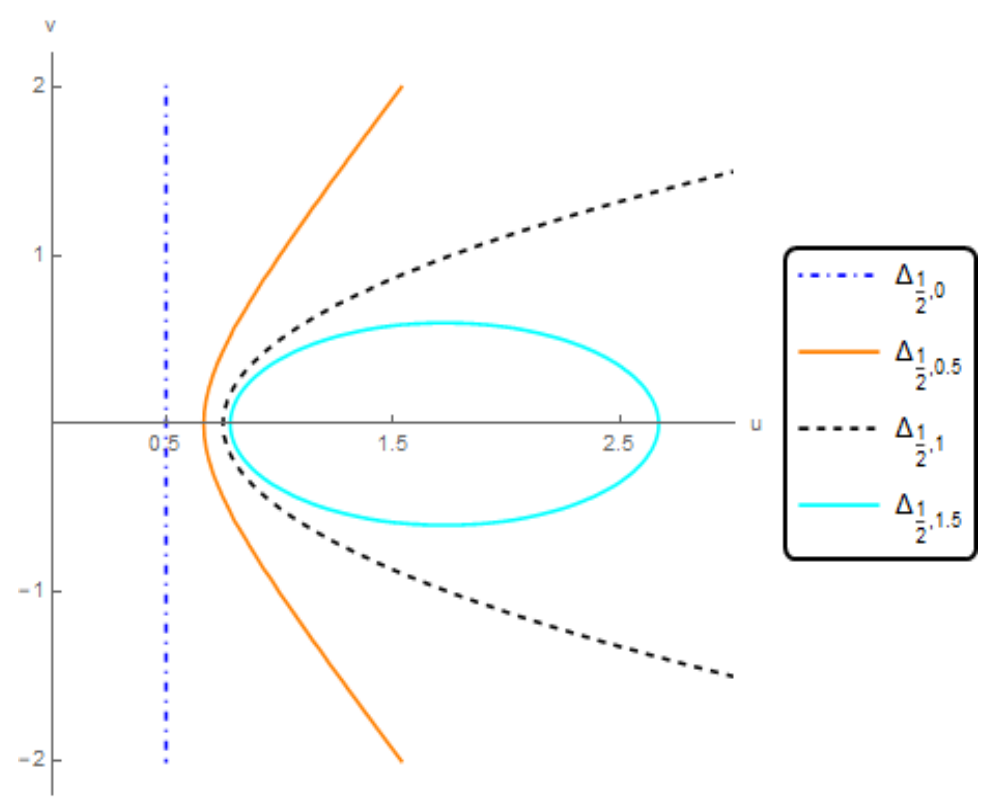

Figure 1: The domain $\triangle_{\alpha, k}=\left\{u+i v:(u-\alpha)^{2}>k^{2}\left((u-1)^{2}+v^{2}\right)\right\}$.

Later on, Aqlan et al. [7 unified the families $\mathcal{U S}(\alpha, k)$ and $\mathcal{U C}(\alpha, k)$ to introduce $\mathcal{U}(k, \alpha, \gamma)$ defined as

$$
\begin{aligned}
\mathcal{U}(k, \alpha, \gamma):=\{f \in \mathcal{A}: & \Re\left(\frac{z f^{\prime}(z)+\gamma z^{2} f^{\prime \prime}(z)}{(1-\gamma) f(z)+\gamma z f^{\prime}(z)}\right) \\
& \left.>k\left|\frac{z f^{\prime}(z)+\gamma z^{2} f^{\prime \prime}(z)}{(1-\gamma) f(z)+\gamma z f^{\prime}(z)}-1\right|+\alpha\right\},
\end{aligned}
$$

where $k \geq 0,0 \leq \alpha<1,0 \leq \gamma \leq 1$. By way of explanation, as $\gamma$ varies from 0 to 1 , this family provides a transition from the class of $k$-uniformly starlike functions of order $\alpha$ and type $\gamma$ to the class of $k$-uniformly convex functions of order $\alpha$ and type $\gamma$ in the open unit disk $\mathbb{D}$. Further generalizations of the above mentioned classes involving complex order also exist in the literature. 


\section{Functions of complex order}

A function $f \in \mathcal{A}$ is said to be starlike of complex order $b(b \in \mathbb{C} \backslash\{0\})$ and type $\alpha(0 \leq \alpha<1)$ if and only if

$$
\Re\left\{1+\frac{1}{b}\left(\frac{z f^{\prime}(z)}{f(z)}-1\right)\right\}>\alpha .
$$

Let us denote the class of all such functions by $\mathcal{S}_{\alpha}^{*}(b)$. Further, a function $f(z)$ of the form (1.1) is said to belong to the class $\mathcal{C}_{\alpha}(b)$ of convex functions of complex order $b(b \in \mathbb{C} \backslash\{0\})$ and type $\alpha(0 \leq \alpha<1)$ if and only if

$$
\Re\left\{1+\frac{1}{b} \frac{z f^{\prime \prime}(z)}{f^{\prime}(z)}\right\}>\alpha .
$$

The function classes $\mathcal{S}_{\alpha}^{*}(b)$ and $\mathcal{C}_{\alpha}(b)$ were introduced and studied by Frasin [16], and the classes $\mathcal{S}_{0}^{*}(b)$ and $\mathcal{C}_{0}(b)$ were considered by Nasr and Aouf 31, 33.

Definition 1.1 (Convolution). Let $f \in \mathcal{A}$ be given by (1.1) and let $g \in \mathcal{A}$ be defined as

$$
g(z)=z+\sum_{n=2}^{\infty} b_{n} z^{n} \quad\left(b_{n} \geq 0\right),
$$

then the convolution (or Hadamard product) of $f$ and $g$, denoted by $f * g$, is defined as

$$
(f * g)(z)=z+\sum_{n=2}^{\infty} a_{n} b_{n} z^{n}=.
$$

Under the operation of Hadamard product, the convex function $p(z)=z /(1-z)$ plays the role of identity element.

In 2008, using the concept of convolution, Raina and Bansal [38] introduced and studied the function class $\mathcal{S}(g, \alpha, k)$ consisting of $f(z)$ satisfying

$$
\Re\left\{\frac{z(f * g)^{\prime}(z)}{(f * g)(z)}-\alpha\right\}>k\left|\frac{z(f * g)^{\prime}(z)}{(f * g)(z)}-1\right|,
$$

for some $g$ given by 1.2 with $(f * g)(z) \neq 0$, where $0 \leq \alpha<1$ and $k \geq 0$. 
To include the uniformly $k$-convex functions of order $\alpha$, Aouf et al. [4, in 2010, extended this class to $\mathcal{S}(g, \gamma, \alpha, k)$ consisting of $f(z)$ satisfying

$$
\begin{aligned}
& \Re\left(\frac{z(f * g)^{\prime}(z)+\gamma z^{2}(f * g)^{\prime \prime}(z)}{(1-\gamma)(f * g)(z)+\gamma z(f * g)^{\prime}(z)}-\alpha\right) \\
> & k\left|\frac{z(f * g)^{\prime}(z)+\gamma z^{2}(f * g)^{\prime \prime}(z)}{(1-\gamma)(f * g)(z)+\gamma z(f * g)^{\prime}(z)}-1\right| .
\end{aligned}
$$

Recently, Bukhari et al. [1] extended the idea of Aouf et al. [4] to define a new class $\mathcal{U M}(g, \gamma, b, k)$ of analytic functions involving complex order as follows:

Definition 1.2. Let the functions $f$ and $g$ be given by (1.1) and (1.2), respectively. Define

$$
\Phi(z):=(f * g)(z) \neq 0, \quad z \in \mathbb{D} .
$$

Then $f \in \mathcal{U M}(g, \gamma, b, k)$, if and only if the following inequality holds:

$\Re\left\{1+\frac{1}{b}\left(\frac{z \Phi^{\prime}(z)+\gamma z^{2} \Phi^{\prime \prime}(z)}{(1-\gamma) \Phi(z)+\gamma z \Phi^{\prime}(z)}-1\right)\right\}>k\left|\frac{1}{b}\left(\frac{z \Phi^{\prime}(z)+\gamma z^{2} \Phi^{\prime \prime}(z)}{(1-\gamma) \Phi(z)+\gamma z \Phi^{\prime}(z)}-1\right)\right|$, where $0 \leq \gamma \leq 1, k \geq 0$, and $b \in \mathbb{C} \backslash\{0\}$.

The authors [11 discussed only a few geometric and analytic properties of functions belonging to the family $\mathcal{U M}(g, \gamma, b, k)$.

Motivated by the above mentioned works, in this paper, we extend the class $\mathcal{U M}(g, \gamma, b, k)$ to the function class $\mathcal{U} \mathcal{M}_{\gamma}(g, b, k, \alpha)$ which consists of analytic functions of complex order $b$ and type $\alpha$. We discuss a number of characteristic and geometric properties of this class and obtain the results of Bukhari et al. 11 as special cases.

Definition 1.3. Let $f \in \mathcal{A}$ be as in (1.1). Then $f \in \mathcal{U M}_{\gamma}(g, b, k, \alpha)$, if for a function $g(z)$ of the form (1.2) satisfying $\Phi(z):=(f * g)(z) \neq 0$, we have

$$
\begin{aligned}
& \Re\left\{(1-\alpha)+\frac{1}{b}\left(\frac{z \Phi^{\prime}(z)+\gamma z^{2} \Phi^{\prime \prime}(z)}{(1-\gamma) \Phi(z)+\gamma z \Phi^{\prime}(z)}-1\right)\right\} \\
> & k\left|\frac{1}{b}\left(\frac{z \Phi^{\prime}(z)+\gamma z^{2} \Phi^{\prime \prime}(z)}{(1-\gamma) \Phi(z)+\gamma z \Phi^{\prime}(z)}-1\right)\right|,
\end{aligned}
$$

where $0 \leq \gamma \leq 1, k \geq 0,0 \leq \alpha<1$ and $b \in \mathbb{C} \backslash\{0\}$. 
We usually call such a class of functions to be defined by the convolution operator $g$. For further details about convolution operators, see Shareef et al. 45] and references therein. For special choices of parameters $\alpha, k, b, \gamma$ and suitable selection of the function $g(z)$, the class $\mathcal{U} \mathcal{M}_{\gamma}(g, b, k, \alpha)$ has been considered earlier. In particular, setting

$$
g(z)=\frac{z}{(1-z)}
$$

and denoting the class $\mathcal{U} \mathcal{M}_{\gamma}\left(\frac{z}{(1-z)}, b, k, \alpha\right)$ by $\mathcal{U} \mathcal{M}_{\gamma}(b, k, \alpha)$, we note that:

(a) The class $\mathcal{U} \mathcal{M}_{\gamma}(b, 0, \alpha)=\mathcal{S C}(b, \gamma, \alpha)$ was considered by Altintas et al. [2].

(b) The class $\mathcal{U M}_{\gamma}(b, 0,0)=\mathcal{P}(\gamma, b)$ was considered by Aouf [3].

(c) $\mathcal{U M}_{\gamma}(1, k, \alpha)=\mathcal{U}(k, \alpha, \gamma)$ was considered by Aqlan et al. [7].

(d) Moreover, the following relationships hold:

$$
\begin{gathered}
\mathcal{U M}_{0}(1, k, \alpha)=\mathcal{U S}^{*}(\alpha, k) \quad \text { and } \quad \mathcal{U M}_{1}(1, k, \alpha)=\mathcal{U C}(\alpha, k), \\
\mathcal{U M}_{0}(b, 0, \alpha)=\mathcal{S}_{b}^{*}(\alpha) \quad \text { and } \quad \mathcal{U M}_{1}(b, 0, \alpha)=\mathcal{C}_{b}(\alpha)
\end{gathered}
$$

For more literature in this direction, we refer to $5,6,12,15,16,28,29,31,34,36,59$.

\section{Functions with negative coefficients}

Let $\mathcal{T}$ denote the subclass of $\mathcal{A}$ consisting of functions with negative coefficients of the form

$$
f(z)=z-\sum_{n=2}^{\infty} a_{n} z^{n} \quad\left(a_{n} \geq 0\right) .
$$

The class $\mathcal{T}$ was first introduced by Silverman [48 and later on studied extensively by a number of authors including the ones in [1,2, 20, 22, 35, 44, 49, 50]. Also see Srivastava et al. 51 57].

The importance of the class $\mathcal{T} \subset \mathcal{A}$ in the theory of univalent functions is due to the fact that some conditions which are only sufficient for the members of $\mathcal{A}$ prove to be both necessary and sufficient for the members of $\mathcal{T}$. The coefficient 
characterization makes several computations in $\mathcal{T}$ manageable which can be very messy and very difficult for the whole of $\mathcal{A}$. For very recent works related to functions with negative coefficients, we refer to $8,10,14,23,30,37,43,46,47$.

Motivated by the above cited works on functions with negative coefficients, in this paper, we study various characteristic properties of the function class $\mathcal{T U M}_{\gamma}(g, b, k, \alpha$ given by

$$
\mathcal{T U M}_{\gamma}(g, b, k, \alpha)=\mathcal{U M}_{\gamma}(g, b, k, \alpha) \cap \mathcal{T}
$$

where $\mathcal{U} \mathcal{M}_{\gamma}(g, b, k, \alpha)$ has been introduced in Definition 1.3. More explicitly, in Section 2, we solve some coefficient problems and determine the radius of close-to-convexity, starlikeness, and convexity for the members of $\mathcal{T U M}_{\gamma}(g, b, k, \alpha)$. Section 3 proves that the family $\mathcal{T U M}_{\gamma}(g, b, k, \alpha)$ is convex and investigates its extreme points. Section 3 also examines the invariance of $\mathcal{T U M}_{\gamma}(g, b, k, \alpha)$ under certain well-known operators, viz, Komato, Bernardi, Libera, etc. In Section 4, we prove that the integral means property holds for the family $\mathcal{T U M}_{\gamma}(g, b, k, \alpha)$. Finally, a subordination problem involving the concept of subordinating factor sequences is discussed in Section 5 .

Note. Now onwards, $f \in \mathcal{A}$ will mean $f(z)$ given in $(1.1)$ and $f \in \mathcal{T}$ will mean $f(z)$ given in (1.5), unless stated otherwise.

\section{Coefficient Estimates and Radii Problems}

The following theorem gives a sufficient condition for of $f \in \mathcal{A}$ to be in $\mathcal{T U}_{\mathcal{\gamma}}(g, b, k, \alpha)$.

Theorem 2.1. Let $f \in \mathcal{A}$ and $g(z)$ be of the form (1.2). If for some $k \geq 0$, $0 \leq \gamma \leq 1,0 \leq \alpha<1$, and $b \in \mathbb{C} \backslash\{0\}$, the inequality

$$
\sum_{n=2}^{\infty}[(k+1)(n-1)+(1-\alpha)|b|][1+\gamma(n-1)]\left|a_{n}\right| b_{n} \leq(1-\alpha)|b|
$$

holds, then $f \in \mathcal{U M}_{\gamma}(g, b, k, \alpha)$. 
Proof. In view of Definition 1.3, $f \in \mathcal{U} \mathcal{M}_{\gamma}(g, b, k, \alpha)$ if

$$
\begin{aligned}
& k\left|\left(\frac{z \Phi^{\prime}(z)+\gamma z^{2} \Phi^{\prime \prime}(z)}{(1-\gamma) \Phi(z)+\gamma z \Phi^{\prime}(z)}-1\right)\right|-\Re\left\{\frac{\bar{b}}{|b|}\left(\frac{z \Phi^{\prime}(z)+\gamma z^{2} \Phi^{\prime \prime}(z)}{(1-\gamma) \Phi(z)+\gamma z \Phi^{\prime}(z)}-1\right)\right\} \\
\leq & (1-\alpha)|b|
\end{aligned}
$$

where the function $\Phi(z)=(f * g)(z)$. For $z$ on the unit circle $\partial \mathbb{D}=\{z:|z|=1\}$, we have

$$
\begin{aligned}
& k\left|\left(\frac{z \Phi^{\prime}(z)+\gamma z^{2} \Phi^{\prime \prime}(z)}{(1-\gamma) \Phi(z)+\gamma z \Phi^{\prime}(z)}-1\right)\right|-\Re\left\{\frac{\bar{b}}{|b|}\left(\frac{z \Phi^{\prime}(z)+\gamma z^{2} \Phi^{\prime \prime}(z)}{(1-\gamma) \Phi(z)+\gamma z \Phi^{\prime}(z)}-1\right)\right\} \\
\leq & (k+1)\left|\left(\frac{z \Phi^{\prime}(z)+\gamma z^{2} \Phi^{\prime \prime}(z)}{(1-\gamma) \Phi(z)+\gamma z \Phi^{\prime}(z)}-1\right)\right| \\
\leq & \quad \sum_{n=2}^{\infty}(n-1)[1+\gamma(n-1)]\left|a_{n}\right| b_{n} \\
& 1-\sum_{n=2}^{\infty}[1+\gamma(n-1)]\left|a_{n}\right| b_{n}
\end{aligned}
$$

This last expression is bounded above by $(1-\alpha)|b|$ if

$$
\sum_{n=2}^{\infty}[(k+1)(n-1)+(1-\alpha)|b|][1+\gamma(n-1)]\left|a_{n}\right| b_{n} \leq(1-\alpha)|b|,
$$

and hence the proof follows by an appeal to maximum modulus theorem.

Remark 1. If we take $\alpha=0$ in Theorem 2.1, we obtain Theorem 3.4 given by Bukhari et al. 11.

The following theorem proves that the condition 2.1) is also necessary for the functions in $\mathcal{T}$.

Theorem 2.2. A necessary and sufficient condition for $f \in \mathcal{T}$ to be in the class $\operatorname{TUM}_{\gamma}(g, b, k, \alpha)$ is that

$$
\sum_{n=2}^{\infty}[(k+1)(n-1)+(1-\alpha)|b|][1+\gamma(n-1)] a_{n} b_{n} \leq(1-\alpha)|b|,
$$

where $k \geq 0,0 \leq \gamma \leq 1,0 \leq \alpha<1$ and $b \in \mathbb{C} \backslash\{0\}$. 
Proof. In view of Theorem 2.1, only necessary part is required to be proven. For $f \in \mathcal{T U M}_{\gamma}(g, b, k, \alpha)$, we have

$$
\begin{aligned}
& \Re\left\{1-\alpha+\frac{1}{b}\left(\frac{z \Phi^{\prime}(z)+\gamma z^{2} \Phi^{\prime \prime}(z)}{(1-\gamma) \Phi(z)+\gamma z \Phi^{\prime}(z)}-1\right)\right\} \\
> & k\left|\frac{1}{b}\left(\frac{z \Phi^{\prime}(z)+\gamma z^{2} \Phi^{\prime \prime}(z)}{(1-\gamma) \Phi(z)+\gamma z \Phi^{\prime}(z)}-1\right)\right|,
\end{aligned}
$$

which further implies that

$$
\begin{gathered}
\Re\left\{1-\alpha-\frac{1}{b}\left(\frac{\sum_{n=2}^{\infty}(n-1)[1+\gamma(n-1)] a_{n} b_{n} z^{n-1}}{1-\sum_{n=2}^{\infty}[1+\gamma(n-1)] a_{n} b_{n} z^{n-1}}\right)\right\} \\
>k\left|\frac{1}{b}\left(\frac{\sum_{n=2}^{\infty}(n-1)[1+\gamma(n-1)] a_{n} b_{n} z^{n-1}}{1-\sum_{n=2}^{\infty}[1+\gamma(n-1)] a_{n} b_{n} z^{n-1}}\right)\right|
\end{gathered}
$$

Letting $z \rightarrow 1^{-}$along the real axis, we have

$$
\begin{gathered}
1-\alpha-\frac{1}{|b|}\left(\frac{\sum_{n=2}^{\infty}(n-1)[1+\gamma(n-1)] a_{n} b_{n}}{1-\sum_{n=2}^{\infty}[1+\gamma(n-1)] a_{n} b_{n}}\right) \\
>k\left[\frac{1}{|b|}\left(\frac{\sum_{n=2}^{\infty}(n-1)[1+\gamma(n-1)] a_{n} b_{n}}{1-\sum_{n=2}^{\infty}[1+\gamma(n-1)] a_{n} b_{n}}\right)\right] .
\end{gathered}
$$

Which on simplification yields the inequality $(2.2)$.

Remark 2. If we fix $b=1$ and $g(z)=z /(1-z)$ in Theorem 2.2, then for $\gamma=0$ we obtain the necessary and sufficient condition for the class $\mathcal{U S}(\alpha, k)$, and for $\gamma=1$, we obtain such conditions for $\mathcal{U C}(\alpha, k)$. 
The following result is an immediate consequence of Theorem 2.2 .

Corollary 2.1. Let $f \in \mathcal{T}$ be in the class $\mathcal{T U M}_{\gamma}(g, b, k, \alpha)$. Then

$$
a_{n} \leq \frac{(1-\alpha)|b|}{[(k+1)(n-1)+(1-\alpha)|b|][1+\gamma(n-1)] b_{n}} \quad(n \geq 2) .
$$

The result is sharp for the function

$$
f(z)=z-\frac{(1-\alpha)|b|}{[(k+1)(n-1)+(1-\alpha)|b|][1+\gamma(n-1)] b_{n}} z^{n} \quad(n \geq 2),
$$

where $k \geq 0,0 \leq \gamma \leq 1,0 \leq \alpha<1$ and $b \in \mathbb{C} \backslash\{0\}$.

\section{Radius Problems}

Definition 2.1 (Radius Problems). Let $\mathcal{F}$ and $\mathcal{G}$ be two subfamilies of $\mathcal{A}$. Then the $\mathcal{F}$-radius of $\mathcal{G}$, denoted by $\mathscr{R}_{\mathcal{F}}(\mathcal{G})$, is the largest number $\rho(0<\rho<1)$ such that $r^{-1} f(r \xi) \in \mathcal{F}$ for all $f \in \mathcal{G}$, where $0<r \leq \rho$. The problem of finding the number $\rho$ is called a radius problem. Further, if we can find an $f_{0} \in \mathcal{G}$ such that $r^{-1} f_{0}(r \xi) \notin \mathcal{F}$ whenever $r>\rho$, then the number $\rho$ is said to be sharp.

Goodman [17, Chapter 13] listed, systematically, several radii results concerning certain classical subfamilies of $\mathcal{S}$.

In the following theorems, we find the radii of close-to-convexity, starlikeness, and convexity for the members of the family $\mathcal{T U M}_{\gamma}(g, b, k, \alpha)$.

A function $f(z)$ analytic in $\mathbb{D}$ is said to belong to the class $\mathcal{K}(\sigma)$ of close-to-convex functions of order $\sigma(0 \leq \sigma<1)$ if there is a convex function $h(z)$ such that $\Re\left\{f^{\prime}(z) / h^{\prime}(z)\right\}>\sigma$. Since $h(z)=z$ is a convex function, it follows that those functions $f(z)$ which satisfy the condition $\Re\left\{f^{\prime}(z)\right\}>\sigma$ are members of the family $\mathcal{K}(\sigma)$.

Theorem 2.3. Let $f \in \mathcal{T U M}_{\gamma}(g, b, k, \alpha)$ be defined by (1.5) and let $g(z)$ be of the form 1.2$)$. Then $f(z)$ is close-to-convex of order $\sigma(0 \leq \sigma<1)$ in the open disk $|z|<r_{c c}$, where

$$
r_{c c}=\inf _{n \geq 2}\left\{\frac{(1-\sigma)[(k+1)(n-1)+(1-\alpha)|b|][1+\gamma(n-1)] b_{n}}{n(1-\alpha)|b|}\right\}^{\frac{1}{n-1}} .
$$

The result is sharp for $f \in \mathcal{T U M}_{\gamma}(g, b, k, \alpha)$ given by 2.3). 
Proof. It is sufficient to prove that $\left|f^{\prime}(z)-1\right| \leq 1-\sigma$ for $|z|<r_{c c}$, where $r_{c c}$ is given by (2.4). From the representation 1.5) of $f(z)$ we have

$$
\left|f^{\prime}(z)-1\right| \leq \sum_{n=2}^{\infty} n a_{n}|z|^{n-1}
$$

Therefore, $\left|f^{\prime}(z)-1\right| \leq 1-\sigma$ if

$$
\sum_{n=2}^{\infty}\left(\frac{n}{1-\sigma}\right) a_{n}|z|^{n-1} \leq 1
$$

Since $f(z) \in \mathcal{T U M}_{\gamma}(g, b, k, \alpha)$, it follows from Theorem 2.2 that

$$
\sum_{n=2}^{\infty} \frac{[(k+1)(n-1)+(1-\alpha)|b|][1+\gamma(n-1)] a_{n} b_{n}}{(1-\alpha)|b|}<1 .
$$

Thus (2.5) will be true if

$$
\left(\frac{n}{1-\sigma}\right)|z|^{n-1} \leq \frac{[(k+1)(n-1)+(1-\alpha)|b|][1+\gamma(n-1)] b_{n}}{(1-\alpha)|b|} .
$$

On further simplification, the above inequality yields the result.

Theorem 2.4. Let the function $f \in \mathcal{T}$ and the function $g(z)$ be defined by 1.2 . Let us suppose that $f(z)$ belongs to the class $\mathcal{T U M}_{\gamma}(g, b, k, \alpha)$. Then

1. $f(z)$ is starlike of order $\sigma(0 \leq \sigma<1)$ in the open disk $|z|<r_{s}$, where

$$
r_{s}=\inf _{n \geq 2}\left\{\frac{(1-\sigma)[(k+1)(n-1)+(1-\alpha)|b|][1+\gamma(n-1)] b_{n}}{(n-\sigma)(1-\alpha)|b|}\right\}^{\frac{1}{n-1}} .
$$

2. $f(z)$ is convex of order $\sigma(0 \leq \sigma<1)$ in the open disk $|z|<r_{c}$, where

$$
r_{c}=\inf _{n \geq 2}\left\{\frac{(1-\sigma)[(k+1)(n-1)+(1-\alpha)|b|][1+\gamma(n-1)] b_{n}}{n(n-\sigma)(1-\alpha)|b|}\right\}^{\frac{1}{n-1}} .
$$

Both the results are sharp. 
Proof. To prove (1), it is sufficient to prove that

$$
\left|\frac{z f^{\prime}(z)}{f(z)}-1\right| \leq 1-\sigma \quad \text { for }|z|<r_{s} .
$$

Indeed, we find from the definition 1.5 of $f(z)$ that

$$
\left|\frac{z f^{\prime}(z)}{f(z)}-1\right| \leq \frac{\sum_{n=2}^{\infty}(n-1) a_{n}|z|^{n-1}}{1-\sum_{n=2}^{\infty} a_{n}|z|^{n-1}} .
$$

Thus

$$
\left|\frac{z f^{\prime}(z)}{f(z)}-1\right| \leq 1-\sigma
$$

if

$$
\sum_{n=2}^{\infty} \frac{(n-\sigma) a_{n}|z|^{n-1}}{1-\sigma} \leq 1
$$

But, by Theorem 2.2, the inequality (2.8) will hold if

$$
\frac{(n-\sigma)|z|^{n-1}}{1-\sigma} \leq \frac{[(k+1)(n-1)+(1-\alpha)|b|][1+\gamma(n-1)] b_{n}}{(1-\alpha)|b|}
$$

that is, if

$$
|z| \leq\left\{\frac{(1-\sigma)[(k+1)(n-1)+(1-\alpha)|b|][1+\gamma(n-1)] b_{n}}{(n-\sigma)(1-\alpha)|b|}\right\}^{\frac{1}{n-1}}, \quad(n \geq 2) .
$$

Hence (1) of Theorem 2.4 follows easily from 2.9). Using the Alexander's characterization that $f(z)$ is convex if and only if $z f^{\prime}(z)$ is starlike, (2) follows easily from (1).

\section{Convexity, Invariance and Extreme Points of $\mathcal{T U M}_{\gamma}(g, b, k, \alpha)$}

A function class $\mathscr{F}$ is said to be convex, if for any $f_{1}, f_{2} \in \mathscr{F}$ and any $\beta \in[0,1]$, one has $\beta f_{1}+(1-\beta) f_{2} \in \mathscr{F}$. 
Theorem 3.1. Let the functions $f_{i}(1 \leq i \leq m ; i \in \mathbb{N})$ be defined as

$$
f_{i}(z)=z-\sum_{n=2}^{\infty} a_{n, i} z^{n} \quad\left(a_{n, i} \geq 0 ; z \in \mathbb{D}\right) .
$$

Suppose that $f_{i} \in \mathcal{T U M}_{\gamma}(g, b, k, \alpha)$ for all $1 \leq i \leq m$. Then the function $h(z)$ given by

$$
h(z)=\sum_{i=1}^{m} \lambda_{i} f_{i}(z) \quad\left(\lambda_{i} \geq 0 ; \sum_{i=1}^{m} \lambda_{i}=1\right),
$$

is also in the class $\operatorname{TUM}_{\gamma}(g, b, k, \alpha)$, where $k \geq 0,0 \leq \gamma \leq 1,0 \leq \alpha<1$ and $b \in \mathbb{C} \backslash\{0\}$.

Proof. Since $f_{i} \in \mathcal{T U M}_{\gamma}(g, b, k, \alpha)$, it follows from Theorem 2.2 that

$$
\sum_{n=2}^{\infty} \frac{[(k+1)(n-1)+(1-\alpha)|b|][1+\gamma(n-1)]}{(1-\alpha)|b|} a_{n, i} b_{n} \leq 1, \quad 1 \leq i \leq m .
$$

From the definition of $h(z)$, we have

$$
h(z)=\sum_{i=1}^{m} \lambda_{i} f_{i}(z)=z-\sum_{n=2}^{\infty}\left(\sum_{i=1}^{m} \lambda_{i} a_{n, i}\right) z^{n}=z-\sum_{n=2}^{\infty} d_{n} z^{n},
$$

where $d_{n}=\sum_{i=1}^{m} \lambda_{i} a_{n, i}, n \geq 2$. Making use of (3.1), we have

$$
\begin{aligned}
& \sum_{n=2}^{\infty} \frac{[(k+1)(n-1)+(1-\alpha)|b|][1+\gamma(n-1)]}{(1-\alpha)|b|} d_{n} b_{n} \\
= & \sum_{i=1}^{m} \lambda_{i}\left\{\sum_{n=2}^{\infty} \frac{[(k+1)(n-1)+(1-\alpha)|b|][1+\gamma(n-1)]}{(1-\alpha)|b|} a_{n, i} b_{n}\right\} \\
\leq & \sum_{i=1}^{m} \lambda_{i}=1 .
\end{aligned}
$$

Therefore, by Theorem 2.2, it follows that $h \in \mathcal{T U M}_{\gamma}(g, b, k, \alpha)$.

Setting $\lambda_{1}=1-\beta, \lambda_{2}=\beta$ and $i=1,2$ in Theorem 3.1 yields

Corollary 3.1. Let the functions $f_{1}, f_{2} \in \mathcal{T U M}_{\gamma}(g, b, k, \alpha)$. Then

$$
(1-\beta) f_{1}(z)+\beta f_{2}(z) \in \mathcal{T U M}_{\gamma}(g, b, k, \alpha) \quad(0 \leq \beta \leq 1) .
$$


We have another result (rotation invariance) related to the class as follows. The proof, being simple, is omitted.

Corollary 3.2. Let $f(z)$ given by (1.5) be in the class $\mathcal{T U M}_{\gamma}(g, b, k, \alpha)$. Then, for $0 \leq \epsilon \leq 1$,

$$
\frac{f(\epsilon z)}{\epsilon} \in \mathcal{T U M}_{\gamma}(g, b, k, \alpha)
$$

Invariance of $\mathcal{T U M}_{\gamma}(g, b, k, \alpha)$ under some well-known operators

In view of Theorem 2.2, it is easy to conclude that if $f \in \mathcal{T U M}_{\gamma}(g, b, k, \alpha)$ is given by 1.5 and

$$
h(z)=z-\sum_{n=2}^{\infty} d_{n} z^{n} \quad\left(d_{n} \geq 0\right)
$$

satisfies $d_{n} \leq a_{n}$ for all $n \geq 2$, then $h \in \mathcal{T U} \mathcal{M}_{\gamma}(g, b, k, \alpha)$.

Theorem 3.2. Let $f \in \mathcal{T U M}_{\gamma}(g, b, k, \alpha)$ be given as

$$
f(z)=z-\sum_{n=2}^{\infty} a_{n} z^{n} \quad\left(a_{n} \geq 0\right),
$$

and let $K_{c}^{p}(z)$ be the Komato operator [26] defined as

$$
K_{c}^{p}[f](z)=\frac{(c+1)^{p}}{\Gamma(p)} \int_{0}^{1}\left(\log \frac{1}{t}\right)^{p-1} t^{c-1} f(t z) d t,
$$

where $c>-1, p \geq 0$, and $\Gamma$ is the well-known gamma function. Then $K_{c}^{p}(z) \in$ $\mathcal{T U M}_{\gamma}(g, b, k, \alpha)$.

Proof. Using the definition of gamma function

$$
\Gamma(z)=\int_{0}^{\infty} t^{z-1} e^{-t} d t \quad(\Re(z)>0),
$$

it is easy to establish that

$$
\frac{(c+1)^{p}}{\Gamma(p)} \int_{0}^{1}\left(\log \frac{1}{t}\right)^{p-1} t^{n+c-1} d t=\left(\frac{c+1}{c+n}\right)^{p} .
$$


Now,

$$
\begin{aligned}
K_{c}^{p}[f](z) & =\frac{(c+1)^{p}}{\Gamma(p)}\left\{z \int_{0}^{1}\left(\log \frac{1}{t}\right)^{p-1} t^{c} d t-\sum_{n=2}^{\infty} a_{n} z^{n} \int_{0}^{1}\left(\log \frac{1}{t}\right)^{p-1} t^{n+c-1} d t\right\} \\
& =z-\sum_{n=2}^{\infty}\left(\frac{c+1}{c+n}\right)^{p} a_{n} z^{n} .
\end{aligned}
$$

For $c>-1$ and $p \geq 0$, it is obvious that

$$
d_{n}=\left(\frac{c+1}{c+n}\right)^{p} a_{n} \leq a_{n} \quad \text { for all } n \geq 2 .
$$

Therefore, it follows that $K_{c}^{p}(z) \in \mathcal{T U}_{\mathcal{\gamma}}(g, b, k, \alpha)$.

Remark 3. For special choices of $c$ and $p$, it follows from Theorem 3.2 that the function class $\mathcal{T U M}_{\gamma}(g, b, k, \alpha)$ is invariant under Alexander, Libera, Bernardi, Jung-Kim-Srivastava operators, see [4].

\section{Extreme Points of $\mathcal{T U} \mathcal{M}_{\gamma}(g, b, k, \alpha)$}

Let $\mathcal{X}$ be a topological vector space over $\mathbb{C}$ and suppose that $U \subset \mathcal{X}$. Then $U$ is convex if $s x_{1}+(1-s) x_{2} \in U$, whenever $x_{1}, x_{2} \in U$ and $s \in(0,1)$. The closed convex hull $H(U)$ of $U$ is the intersection of all closed convex sets containing $U$. A point $u \in U \subset \mathcal{X}$ is said to be an extreme point of $U$ if it can not be written as $u=s x+(1-s) y$ for distinct $x_{1}, x_{2} \in U$ and $0<s<1$.

Since the family $\mathcal{T U}_{p}^{n}(g, \gamma, k, b, \alpha)$ is convex, we determine its extereme points.

Theorem 3.3. Let $f_{1}(z)=z$ and

$$
f_{n}(z)=z-\frac{(1-\alpha)|b|}{[(k+1)(n-1)+(1-\alpha)|b|][1+\gamma(n-1)] b_{n}} z^{n} \quad(n \geq 2),
$$

for $0 \leq \gamma \leq 1,0 \leq \alpha<1, k \geq 0$ and $b \in \mathbb{C} \backslash\{0\}$. Then $f(z)$ is in the class $\mathcal{T U M}_{\gamma}(g, b, k, \alpha)$ if and only if it can be expressed as

$$
f(z)=\sum_{n=1}^{\infty} \lambda_{n} f_{n}(z),
$$

where $\lambda_{n} \geq 0$ and $\sum_{n=1}^{\infty} \lambda_{n}=1$. 
Proof. Suppose that $f(z)$ can be expressed as (3.3). We show that $f \in$ $\mathcal{T U M}_{\gamma}(g, b, k, \alpha)$. From (3.3), we have that

$$
\begin{aligned}
f(z) & =\sum_{n=1}^{\infty} \lambda_{n} f_{n}(z) \\
& =\lambda_{1} f_{1}(z)+\sum_{n=2}^{\infty} \lambda_{n} f_{n}(z) \\
& =z-\sum_{n=2}^{\infty} \lambda_{n} \frac{(1-\alpha)|b|}{[(k+1)(n-1)+(1-\alpha)|b|][1+\gamma(n-1)] b_{n}} z^{n} \\
& =z-\sum_{n=2}^{\infty} \Psi_{n} z^{n}
\end{aligned}
$$

where

$$
\Psi_{n}=\lambda_{n} \frac{(1-\alpha)|b|}{[(k+1)(n-1)+(1-\alpha)|b|][1+\gamma(n-1)] b_{n}} .
$$

Now,

$$
\begin{aligned}
\sum_{n=2}^{\infty}[(k+1)(n-1)+(1-\alpha)|b|][1+\gamma(n-1)] b_{n} \Psi_{n} & =\sum_{n=2}^{\infty} \lambda_{n}(1-\alpha)|b| \\
& =(1-\alpha)|b|\left[1-\lambda_{1}\right] \\
& \leq(1-\alpha)|b| .
\end{aligned}
$$

Therefore, $f(z)$ is in the class $\operatorname{TUM}_{\gamma}(g, b, k, \alpha)$ follows by Theorem 2.2 Conversely, assume that $f(z)$ defined by 1.5 belongs to the class $\mathcal{T U M}_{\gamma}(g, b, k, \alpha)$. Then

$$
a_{n} \leq \frac{(1-\alpha)|b|}{[(k+1)(n-1)+(1-\alpha)|b|][1+\gamma(n-1)] b_{n}} \quad(n \geq 2) .
$$

Setting

$$
\lambda_{n}=\frac{[(k+1)(n-1)+(1-\alpha)|b|][1+\gamma(n-1)] a_{n} b_{n}}{(1-\alpha)|b|} \quad(n \geq 2)
$$

and $\lambda_{1}=1-\sum_{n=2}^{\infty} \lambda_{n}$, we can see that $f(z)$ can be expressed in the form 3.3 . 
Corollary 3.3. The extreme points of the class $\operatorname{TUM}_{\gamma}(g, b, k, \alpha)$ are the functions $f_{1}(z)=z$ and

$$
f_{n}(z)=z-\frac{(1-\alpha)|b|}{[(k+1)(n-1)+(1-\alpha)|b|][1+\gamma(n-1)] b_{n}} z^{n} \quad(n \geq 2) .
$$

\section{Integral Means Property}

Baernstein [9] proved that for $f \in \mathcal{S}$ and the Koebe function $k(z)=z /(1-z)^{2}$,

$$
\int_{0}^{2 \pi}\left|f\left(r e^{i \theta}\right)\right|^{\mu} d \theta \leq \int_{0}^{2 \pi}\left|k\left(r e^{i \theta}\right)\right|^{\mu} d \theta \quad\left(z=r e^{i \theta}\right)
$$

for all $0<r<1$ and $\mu>0$. Silverman 48 found that the function $\widehat{f}(z)=z-z^{2} / 2$ often serves as an extremal function in $\mathcal{T}$ as the Koebe function $k(z)=z /(1-z)^{2}$ does in $\mathcal{S}$. Silverman [49, Open Problem 9] conjectured that: For $f \in \mathcal{T}$, is it true that

$$
\int_{0}^{2 \pi}\left|f\left(r e^{i \theta}\right)\right|^{\mu} d \theta \leq \int_{0}^{2 \pi}\left|\widehat{f}\left(r e^{i \theta}\right)\right|^{\mu} d \theta \quad \forall r<1, \mu>0 ?
$$

He himself settled this conjecture in [50]. In this section we prove Silverman's conjecture for functions belonging to the class $\mathcal{T U M}_{\gamma}(g, b, k, \alpha)$. To prove our result we need the following information.

Definition 4.1 (Subordination). Let $f$ and $g$ be analytic in $\mathbb{D}$. Then we say that $f$ is subordinate to $g(f \prec g)$ if there exists a function $w$, analytic in $\mathbb{D}$ with $w(0)=0$ and $|w(z)|<1$, such that $f(z)=g(w(z))$ for all $z \in \mathbb{D}$. Furthermore, if the function $g(z)$ is univalent in $\mathbb{D}$, then $f \prec g$ if and only if

$$
f(0)=g(0) \text { and } f(\mathbb{D}) \subset g(\mathbb{D}) .
$$

Lemma 4.1 (Littlewood, 27]). Let the functions $f(z)$ and $g(z)$ be analytic in $\mathbb{D}$ and let $f \prec g$. Then

$$
\int_{0}^{2 \pi}\left|f\left(r e^{i \theta}\right)\right|^{\mu} d \theta \leq \int_{0}^{2 \pi}\left|g\left(r e^{i \theta}\right)\right|^{\mu} d \theta, \quad z=r e^{i \theta}, \mu>0,0<r<1 .
$$


Theorem 4.1. Let $f \in \mathcal{T U M}_{\gamma}(g, b, k, \alpha)$ be of the form 1.5), where $0 \leq \gamma \leq$ $1,0 \leq \alpha<1, k \geq 0$ and $b \in \mathbb{C} \backslash\{0\}$. Define $f_{2}(z)$ by

$$
f_{2}(z)=z-\frac{(1-\alpha)|b|}{[k+1+(1-\alpha)|b|][1+\gamma] b_{2}} z^{2} .
$$

Then for $\mu>0$ and $z=r e^{i \theta}(0<r<1)$, we have

$$
\int_{0}^{2 \pi}\left|f\left(r e^{i \theta}\right)\right|^{\mu} d \theta \leq \int_{0}^{2 \pi}\left|f_{2}\left(r e^{i \theta}\right)\right|^{\mu} d \theta .
$$

Proof. In view of (1.5), proving (4.1) is equivalent to prove

$$
\int_{0}^{2 \pi}\left|1-\sum_{n=2}^{\infty} a_{n} z^{n-1}\right|^{\mu} d \theta \leq \int_{0}^{2 \pi}\left|1-\frac{(1-\alpha)|b|}{[k+1+(1-\alpha)|b|][1+\gamma] b_{2}} z\right|^{\mu} d \theta
$$

By Lemma 4.1, the result will follow if we show

$$
1-\sum_{n=2}^{\infty} a_{n} z^{n-1} \prec 1-\frac{(1-\alpha)|b|}{[k+1+(1-\alpha)|b|][1+\gamma] b_{2}} z .
$$

Setting

$$
1-\sum_{n=2}^{\infty} a_{n} z^{n-1}=1-\frac{(1-\alpha)|b|}{[k+1+(1-\alpha)|b|][1+\gamma] b_{2}} w(z) .
$$

This implies

$$
w(z)=\sum_{n=2}^{\infty} \frac{[k+1+(1-\alpha)|b|][1+\gamma] b_{2}}{(1-\alpha)|b|} a_{n} z^{n-1} \text { with } w(0)=0 .
$$

Using (2.2), we obtain

$$
\begin{aligned}
|w(z)| & =\left|\sum_{n=2}^{\infty} \frac{[k+1+(1-\alpha)|b|][1+\gamma] b_{2}}{(1-\alpha)|b|} a_{n} z^{n-1}\right| \\
& \leq|z| \sum_{n=2}^{\infty} \frac{[k+1+(1-\alpha)|b|][1+\gamma] b_{2}}{(1-\alpha)|b|} a_{n} \\
& \leq|z| \sum_{n=2}^{\infty} \frac{[(k+1)(n-1)+(1-\alpha)|b|][1+\gamma(n-1)] b_{n}}{(1-\alpha)|b|} a_{n} \\
& \leq|z|<1 .
\end{aligned}
$$

This establishes 4.2 and hence completes the proof. 


\section{Subordination Theorem}

Definition 5.1 (Subordinating Factor Sequence). A sequence $\left\{s_{n}\right\}_{n=1}^{\infty}$ of complex numbers is said to be a subordinating factor sequence if for every convex univalent function

$$
\Psi(z)=z+\sum_{n=2}^{\infty} c_{n} z^{n} \quad(z \in \mathbb{D})
$$

we have the subordination given by

$$
\sum_{n=1}^{\infty} s_{n} c_{n} z^{n} \prec \Psi(z) \quad\left(z \in \mathbb{D} ; c_{1}=1\right) .
$$

The following criterion for a sequence of complex numbers $\left\{s_{n}\right\}_{n=1}^{\infty}$ to be a subordinating factor sequence was established by Wilf [58].

Lemma 5.1 ( [58, Theorem 2]). The sequence $\left\{s_{n}\right\}_{n=1}^{\infty}$ is a subordinating factor sequence if and only if

$$
\Re\left\{1+2 \sum_{n=1}^{\infty} s_{n} z^{n}\right\}>0 .
$$

For further results related to subordinating factor sequence, refer Raina and Bansal [38] and Ruscheweyh [42].

Theorem 5.1. Let the function $f \in \mathcal{A}$ satisfies the condition (2.1) and let

$$
v=\frac{[k+1+(1-\alpha)|b|](1+\gamma) b_{2}}{2\left\{[k+1+(1-\alpha)|b|](1+\gamma) b_{2}+(1-\alpha)|b|\right\}} .
$$

Then for every convex function $\Psi(z)$ we have

$$
v(f * q)(z) \prec \Psi(z) \quad(z \in \mathbb{D}) .
$$

Furthermore,

$$
\Re\{f(z)\}>-\frac{1}{2 v} \quad(z \in \mathbb{D}) .
$$

The constant factor $v$ in $(5.2)$ and (5.3) is best possible. 
Proof. Let $\Psi(z)$ be defined by (5.1). Then

$$
v(f * q)(z)=\sum_{n=1}^{\infty} s_{n} c_{n} z^{n}
$$

where $c_{1}=1$ and

$$
s_{n}= \begin{cases}v, & \text { if } n=1 \\ v a_{n}, & \text { if } n \geq 2 .\end{cases}
$$

In view of Definition 5.1 and Lemma 5.1, the subordination 5.2 will hold true if

$$
\Re\left\{1+2 \sum_{n=1}^{\infty} s_{n} z^{n}\right\}>0 .
$$

Since

$$
[k+1+(1-\alpha)|b|](1+\gamma) b_{2} \leq[(k+1)(n-1)+(1-\alpha)|b|][1+\gamma(n-1)] b_{n} \quad(n \geq 2),
$$

we have for $|z|=r<1$,

$$
\begin{aligned}
\Re\left\{1+2 \sum_{n=1}^{\infty} s_{n} z^{n}\right\} \geq & 1-2 v r-2 \sum_{n=2}^{\infty} v\left|a_{n}\right| r^{n} \\
\geq 1 & -\frac{[k+1+(1-\alpha)|b|](1+\gamma) b_{2}}{\left\{[k+1+(1-\alpha)|b|](1+\gamma) b_{2}+(1-\alpha)|b|\right\}} r \\
& \quad-\frac{\sum_{n=2}^{\infty}[(k+1)(n-1)+(1-\alpha)|b|][1+\gamma(n-1)]\left|a_{n}\right| b_{n} r^{n}}{2\left\{[k+1+(1-\alpha)|b|](1+\gamma) b_{2}+(1-\alpha)|b|\right\}} .
\end{aligned}
$$

Which on using (2.1) gives

$$
\Re\left\{1+2 \sum_{n=1}^{\infty} s_{n} z^{n}\right\} \geq 1-r>0 .
$$

Hence the subordination result $(5.2)$ is proved. Now, letting $\Psi(z)=z /(1-z)=$ $z+\sum_{n=2}^{\infty} z^{n}$ in 5.2 and noting that this function maps $\mathbb{D}$ onto the half-plane $\Re(\Psi(z))>-1 / 2$, the result $(5.3)$ follows easily. We now prove the sharpness of $v$. Consider the function

$$
f_{2}(z)=z-\frac{(1-\alpha)|b|}{[k+1+(1-\alpha)|b|](1+\gamma) b_{2}} z^{2}
$$


This function satisfies the condition (2.1), and hence we have from 5.2

$$
v f_{2}(z) \prec \frac{z}{1-z} .
$$

Moreover, it can be easily verified that $\min _{|z| \leq r} \Re\left\{f_{2}(z)\right\}=-1 / 2 v$. Therefore, the constant $v$ cannot be replaced by a larger number.

Remark 4. Taking $\alpha=0$ in Theorem 5.1, we get the subordination result obtained by Bukhari et al. [11, Theorem 3.4]

Taking $\gamma=0$, we have

Corollary 5.1. Let $f(z) \in \mathcal{A}$ satisfy

$$
\sum_{n=2}^{\infty}[(k+1)(n-1)+(1-\alpha)|b|]\left|a_{n}\right| b_{n} \leq(1-\alpha)|b| .
$$

Then for every convex function $\Psi(z)$ we have

$$
\frac{[k+1+(1-\alpha)|b|] b_{2}}{2\left[(k+1) b_{2}+(1-\alpha)\left(b_{2}+1\right)|b|\right]}(f * \Psi)(z) \prec \Psi(z)
$$

and

$$
\Re\{f(z)\}>-\frac{\left[(k+1) b_{2}+(1-\alpha)\left(b_{2}+1\right)|b|\right]}{[k+1+(1-\alpha)|b|] b_{2}} \quad(z \in \mathbb{D}) .
$$

The constant factor $\frac{[k+1+(1-\alpha)|b|] b_{2}}{2\left[(k+1) b_{2}+(1-\alpha)\left(b_{2}+1\right)|b|\right]}$ in 5.4 is the best possible.

Taking $\gamma=1$ yields

Corollary 5.2. Let $f \in \mathcal{A}$ satisfy

$$
\sum_{n=2}^{\infty} n[(k+1)(n-1)+(1-\alpha)|b|]\left|a_{n}\right| b_{n} \leq(1-\alpha)|b| .
$$

Then for every convex function $\Psi(z)$ we have

$$
\frac{[k+1+(1-\alpha)|b|] b_{2}}{\left[2(k+1) b_{2}+(1-\alpha)\left(2 b_{2}+1\right)|b|\right]}(f * \Psi)(z) \prec \Psi(z)
$$

and

$$
\Re\{f(z)\}>-\frac{\left[2(k+1) b_{2}+(1-\alpha)\left(2 b_{2}+1\right)|b|\right]}{[k+1+(1-\alpha)|b|] b_{2}} \quad(z \in \mathbb{D}) .
$$

The constant factor $\frac{[k+1+(1-\alpha)|b|] b_{2}}{\left[2(k+1) b_{2}+(1-\alpha)\left(2 b_{2}+1\right)|b|\right]}$ is best possible. 
Taking $k=0, \gamma=0$, the following result was obtained by Prajapat 36 .

Corollary 5.3 ( $[36$, Theorem 3.1]). Let $f \in \mathcal{A}$ satisfy

$$
\sum_{n=2}^{\infty}[n-1+(1-\alpha)|b|]\left|a_{n}\right| b_{n} \leq(1-\alpha)|b| .
$$

Then for every convex function $\Psi(z)$ we have

$$
\frac{[1+(1-\alpha)|b|] b_{2}}{2\left[b_{2}+(1-\alpha)\left(b_{2}+1\right)|b|\right]}(f * \Psi)(z) \prec \Psi(z)
$$

and

$$
\Re\{f(z)\}>-\frac{\left[b_{2}+(1-\alpha)\left(b_{2}+1\right)|b|\right]}{[1+(1-\alpha)|b|] b_{2}} \quad(z \in \mathbb{D}) .
$$

The constant factor $\frac{[1+(1-\alpha)|b|] b_{2}}{2\left[b_{2}+(1-\alpha)\left(b_{2}+1\right)|b|\right]}$ cannot be replaced by a larger one.

\section{Concluding Remarks}

Remark 5. Setting $b=1$ and then specializing the function $g(z)$ and the parameters $\gamma, k, \alpha$ in the class $\mathcal{T U M}_{\gamma}(g, b, k, \alpha)$, all the theory related to the class considered by Aouf et al. [4] and the classes they have mentioned ( [4, item (i)-(viii)]) can be obtained easily.

Remark 6. The class can be extended to the class of functions which are $p$-valent in $\mathbb{D}$ and hence generalizing a number of results available in the literature.

\section{References}

[1] O. P. Ahuja and P. K. Jain, On starlike and convex functions with missing and negative coefficients, Bull. Malaysian Math. Soc. (2) 3(2) (1980), 95-101.

[2] O. Altintas, Ö. Ozkan and H. M. Srivastava, Neighborhoods of a class of analytic functions with negative coefficients, Appl. Math. Lett. 13(3) (2000), 63-67. https://doi.org/10.1016/S0893-9659(99)00187-1

[3] M. K. Aouf, Some subordinations results for certain subclasses of starlike and convex functions of complex order, Acta Univ. Apulensis Math. Inform. 35 (2013), 101-110. 
[4] M. K. Aouf, R. M. El-Ashwah and S. M. El-Deeb, Certain subclasses of uniformly starlike and convex functions defined by convolution, Acta Math. Acad. Paedagog. Nyházi. (N.S.) 26(1) (2010), 55-70.

[5] M. K. Aouf et al., Subordination results for certain class of analytic functions defined by convolution, Rend. Circ. Mat. Palermo (2) 60(1-2) (2011), 255-262. https://doi.org/10.1007/s12215-011-0048-0

[6] M. K. Aouf et al., Subordination theorem of analytic functions defined by convolution, Complex Anal. Oper. Theory 7(4) (2013), 1117-1126. https://doi.org/10.1007/s11785-011-0171-0

[7] E. Aqlan, J.M. Jahangiri and S.R. Kulkarni, New classes of $k$-uniformly convex and starlike functions, Tamkang J. Math. 35(3) (2004), 261-266. https://doi.org/10.5556/j.tkjm.35.2004.207

[8] W. G. Atshan, A. K. Wanas and G. Murugusundaramoorthy, Properties and characteristics of certain subclass of multivalent prestarlike functions with negative coefficients, An. Univ. Oradea Fasc. Mat. 26(2) (2019), 17-24.

[9] A. Baernstein, II, Integral means, univalent functions and circular symmetrization, Acta Math. 133 (1974), 139-169. https://doi.org/10.1007/BF02392144

[10] R. Bucur and D. Breaz, Properties of a new subclass of analytic functions with negative coefficients defined by using the $q$-derivative, Appl. Math. Nonlinear Sci. 5(1) (2020), 303-308. https://doi.org/10.2478/amns.2020.1.00028

[11] S. Z. H. Bukhari, J. Sokol and S. Zafar, Unified approach to starlike and convex functions involving convolution between analytic functions, Results Math. 73(1) (2018), Art. 30, 12 pp. https://doi.org/10.1007/s00025-018-0782-0

[12] Q. Deng, Certain subclass of analytic functions with complex order, Appl. Math. Comput. 208(2) (2009), 359-362. https://doi.org/10.1016/j.amc.2008.12.018

[13] E. Deniz, H. Orhan and J. Sokół, Classes of analytic functions defined by a differential operator related to conic domains, Ukrainian Math. J. 67(9) (2016), 1367-1385. https://doi.org/10.1007/s11253-016-1159-8

[14] K. K. Dixit et al., On a new class of fractional operator associated with $k$-uniformly convex functions with negative coefficients, Ganita 70(2) (2020), 193-199. 
[15] J. Dziok and H. M. Srivastava, Classes of analytic functions associated with the generalized hypergeometric function, Applied Mathematics and Computation 103(1) (1999), 1-13. https://doi.org/10.1016/S0096-3003(98)10042-5

[16] B. A. Frasin, Family of analytic functions of complex order, Acta Math. Acad. Paedagog. Nyházi.(NS) 22(2) (2006), 179-191.

[17] A. W. Goodman, Univalent Functions, Vol. II, Mariner Publishing Co., Inc., Tampa, FL, 1983.

[18] A. W. Goodman, On uniformly convex functions, Ann. Polon. Math. 56(1) (1991), 87-92. https://doi.org/10.4064/ap-56-1-87-92

[19] A. W. Goodman, On uniformly starlike functions, J. Math. Anal. Appl. 155(2) (1991), 364-370. https://doi.org/10.1016/0022-247X(91)90006-L

[20] V. P. Gupta and P. K. Jain, Certain classes of univalent functions with negative coefficients, Bull. Austral. Math. Soc. 14(3) (1976), 409-416.

https://doi.org/10.1017/S0004972700025326

[21] V. P. Gupta and P. K. Jain, Certain classes of univalent functions with negative coefficients. II, Bull. Austral. Math. Soc. 15(3) (1976), 467-473.

https://doi.org/10.1017/S0004972700022917

[22] P. K. Jain and O. P. Ahuja, A class of univalent functions with negative coefficients, Rend. Mat. (7) 1(1) (1981), 47-54.

[23] S. B. Joshi, S. S. Joshi and H. Pawar, Applications of generalized fractional integral operator to unified subclass of prestarlike functions with negative coefficients, Stud. Univ. Babeş-Bolyai Math. 63(1) (2018), 59-69.

https://doi.org/10.24193/subbmath.2018.1.04

[24] S. Kanas and A. Wisniowska, Conic regions and $k$-uniform convexity, J. Comput. Appl. Math. 105(1-2) (1999), 327-336.

https://doi.org/10.1016/S0377-0427(99)00018-7

[25] S. Kanas and A. Wiśniowska, Conic regions and k-uniform convexity. II, Zeszyty Nauk. Politech. Rzeszowskiej Mat. 170 (1998), 65-78.

[26] Y. Komatu, On analytic prolongation of a family of operators, Mathematica (Cluj) $32(55)$ (1990), no. 2, 141-145. 
[27] J. E. Littlewood, On inequalities in the theory of functions, Proc. London Math. Soc. (2) 23(7) (1925), 481-519. https://doi.org/10.1112/plms/s2-23.1.481

[28] G. Murugusundaramoorthy and H. M. Srivastava, Neighborhoods of certain classes of analytic functions of complex order, JIPAM. J. Inequal. Pure Appl. Math. 5(2) (2004), Article 24, 8 pp.

[29] G. Murugusundaramoorthy and N. Magesh, Starlike and convex functions of complex order involving the Dziok-Srivastava operator, Integral Transforms Spec. Funct. 18(5-6) (2007), 419-425. https://doi.org/10.1080/10652460701318111

[30] N. Mustafa and O. Altintaş, Normalized Wright functions with negative coefficients and some of their integral transforms, TWMS J. Pure Appl. Math. 9(2) (2018), 190-206.

[31] M. A. Nasr and M. K. Aouf, Bounded starlike functions of complex order, Proc. Indian Acad. Sci. Math. Sci. 92(2) (1983), 97-102.

https://doi.org/10.1007/BF02863012

[32] M. A. Nasr and M. K. Aouf, Radius of convexity for the class of starlike functions of complex order, Bull. Fac. Sci. Assiut Univ. A 12(1) (1983), 153-159.

[33] M. A. Nasr and M. K. Aouf, Starlike function of complex order, J. Natur. Sci. Math. 25(1) (1985), 1-12.

[34] K. I. Noor, M. Arif and W. Ul-Haq, On $k$-uniformly close-to-convex functions of complex order, Appl. Math. Comput. 215(2) (2009), 629-635.

https://doi.org/10.1016/j.amc.2009.05.050

[35] S. Owa and M. Obradović, New classification of analytic functions with negative coefficients, Internat. J. Math. Math. Sci. 11(1) (1988), 55-69.

https://doi.org/10.1155/S0161171288000109

[36] J. K. Prajapat, Subordination theorem for a family of analytic functions associated with the convolution structure, Journal of Inequalities in Pure and Applied Mathematics 9(4) (2008), Article 102, 8 pp.

[37] A. H. El-Qadeem and M. A. Mamon, Comprehensive subclasses of multivalent functions with negative coefficients defined by using a q-difference operator, Trans. A. Razmadze Math. Inst. 172(3) Part B (2018), 510-526.

https://doi.org/10.1016/j.trmi.2018.04.002 
[38] R. K. Raina and D. Bansal, Some properties of a new class of analytic functions defined in terms of a Hadamard product, JIPAM. J. Inequal. Pure Appl. Math. 9(1) (2008), Article 22, 9 pp.

[39] M. I. Robertson, On the theory of univalent functions, Annals of Mathematics 37(2) (1936), 374-408. https://doi.org/10.2307/1968451

[40] F. Ronning, On starlike functions associated with parabolic regions, Ann. Univ. Mariae Curie-Sktodowska Sect. A 45 (1991), 117-122 (1992).

[41] F. Ronning, Uniformly convex functions and a corresponding class of starlike functions, Proc. Amer. Math. Soc. 118(1) (1993), 189-196.

https://doi.org/10.2307/2160026

[42] S. Ruscheweyh, New criteria for univalent functions, Proc. Amer. Math. Soc. 49 (1975), 109-115. https://doi.org/10.1090/S0002-9939-1975-0367176-1

[43] G. S. Sălăgean and A. Venter, On the order of convolution consistence of the analytic functions with negative coefficients, Math. Bohem. 142(4) (2017), 381-386. https://doi.org/10.21136/MB.2017.0019-15

[44] S. M. Sarangi and B. A. Uralegaddi, The radius of convexity and starlikeness for certain classes of analytic functions with negative coefficients. II, Atti. Acad. Naz. Lincci Rend. Sc. Fis. Mat. Natur. (8) 67(1-2) (1979), 16-20 (1980).

[45] Z. Shareef, S. Hussain and M. Darus, Convolution operators in the geometric function theory, J. Inequal. Appl. 2012 (2012), Article No. 213, 11 pp. https://doi.org/10.1186/1029-242X-2012-213

[46] N. Shilpa, Some properties of subclasses of analytic functions with negative coefficients, South East Asian J. Math. Math. Sci. 15(3) (2019), 41-51.

[47] A. S. Shinde et al., A certain subclass of uniformly convex functions with negative coefficients defined by Caputo's fractional calculus operator, J. Fract. Calc. Appl. 12(1) (2021), 172-183.

[48] H. Silverman, Univalent functions with negative coefficients, Proc. Amer. Math. Soc. 51 (1975), 109-116. https://doi.org/10.1090/S0002-9939-1975-0369678-0 
[49] H. Silverman, A survey with open problems on univalent functions whose coefficients are negative, Rocky Mountain J. Math. 21(3) (1991), 1099-1125.

https://doi.org/10.1216/rmjm/1181072932

[50] H. Silverman, Integral means for univalent functions with negative coefficients, Houston J. Math. 23(1) (1997), 169-174.

[51] H. M. Srivastava and M. K. Aouf, Some applications of fractional calculus operators to certain subclasses of prestarlike functions with negative coefficients, Comput. Math. Appl. 30(1) (1995), 53-61.

https://doi.org/10.1016/0898-1221(95)00067-9

[52] H. M. Srivastava, H. M. Hossen and M. K. Aouf, A certain subclass of meromorphically convex functions with negative coefficients, Math. J. Ibaraki Univ. 30 (1998), 33-51. https://doi.org/10.5036/mjiu.30.33

[53] H. M. Srivastava, A. K. Mishra and M. K. Das, A unified operator in fractional calculus and its applications to a nested class of analytic functions with negative coefficients, Complex Variables Theory Appl. 40(2) (1999), 119-132.

https://doi.org/10.1080/17476939908815211

[54] H. M. Srivastava, S. Owa and K. Nishimoto, Certain subclasses of functions of positive real part with negative coefficients, J. College Engrg. Nihon Univ. Ser. B 27 (1986), 47-55.

[55] H. M. Srivastava, J. Patel and P. Sahoo, Some families of analytic functions with negative coefficients, Math. Slovaca 51(4) (2001), 421-439.

[56] H. M. Srivastava et al., A new subclass of $k$-uniformly convex functions with negative coefficients, JIPAM. J. Inequal. Pure Appl. Math. 8(2) (2007), Article 43, 14 pp.

[57] H. M. Srivastava, S. Sümer Eker and B. Şeker, A certain convolution approach for subclasses of analytic functions with negative coefficients, Integral Transforms Spec. Funct. 20(9-10) (2009), 687-699. https://doi.org/10.1080/10652460902749437

[58] H. S. Wilf, Subordinating factor sequences for convex maps of the unit circle, Proc. Amer. Math. Soc. 12 (1961), 689-693.

https://doi.org/10.1090/S0002-9939-1961-0125214-5 
[59] L. Zhou and Q. Xu, Coefficient estimates for certain subclasses of analytic functions of complex order, Eur. J. Pure Appl. Math. 6(4) (2013), 460-468.

This is an open access article distributed under the terms of the Creative Commons Attribution License (http://creativecommons.org/licenses/by/4.0/), which permits unrestricted, use, distribution and reproduction in any medium, or format for any purpose, even commercially provided the work is properly cited. 